\title{
Tuberculosis Diagnostics and Susceptibility in Saudi Arabia's Western Region
}

\author{
${ }^{1}$ Mohammed Qutub, ${ }^{2}$ Ghassan Wali, ${ }^{3}$ Basem Alraddadi, ${ }^{4}$ Nezar Bahabri, ${ }^{5}$ Yasser Aldabbagh ${ }^{*},{ }^{6}$ Sara \\ Zaki, ${ }^{7}$ Fahtima Mehdawi, ${ }^{8}$ Prasanth Govindan, ${ }^{9}$ Anupama Vattappillil, ${ }^{10}$ Ebtihal Alamoudi \\ ${ }^{1,8,9}$ Department of Pathology and Laboratory Medicine, King Faisal Specialist Hospital and Research Center, \\ Jeddah, Saudi Arabia \\ ${ }^{2,3}$ Department of Internal Medicine, King Faisal Specialist Hospital and Research Center, Jeddah, Saudi \\ Arabia \\ ${ }^{4,5,6,7}$ Department of Internal Medicine, Dr. Soliman Fakeeh Hospital, Jeddah, Saudi Arabia \\ ${ }^{10}$ Department of Microbiology, King Saud University Medical City, Riyadh, Saudi Arabia
}

\begin{abstract}
Introduction: Saudi Arabia has a moderate infection rate of tuberculosis (TB) in comparison to other countries in the region. The prevalence of TB varies among different parts of the Kingdom, with its western provinces reporting to have the leading rate of incidence.

Method: In this retrospective study, Acid-fast bacilli smear (AFB), and nucleic acid amplification test (NAAT) were performed on all positive cultures from 254 identified TB cases from November 2006 to August 2016 in King Faisal Specialist Hospital and Research Center - Jeddah, Saudi Arabia.

Results: From the positive cultures for Mycobacterium tuberculosis complex, $99.1 \%$ were found to be positive by using NAAT. From the NAAT positive samples, only $36.8 \%$ were positive for AFB. TB was predominant among the younger age group (59.4\%) compared to the elderly population (35.8\%) and pediatrics $(4.7 \%)$, and it was also observed that males had a higher prevalence of $56.3 \%$ in comparison to females. In regards to the site of infection, $53.1 \%$ were pulmonary and $46.9 \%$ from an extrapulmonary. It was noted that resistance to first-line anti-TB drugs individually was found to be $11.0 \%$ to Streptomycin, $10.2 \%$ to isoniazid, $5.1 \%$ to pyrazinamide, $2.4 \%$ to Rifampicin, and $1.2 \%$ Ethambutol, yet multi-drug resistance (MDR) was found in $2.4 \%$ of observed cases.

Conclusion: Young age predominance, low direct smear positivity, increased incidence of the extrapulmonary site of infection, and re-emergence of TB resistance all were observed in our study compared to previous national surveys.
\end{abstract}

Keywords: Tuberculosis (TB), acid fact bacilli (AFB), Nucleic acid amplification test (NAAT), Multidrug resistant tuberculosis (MDR-TB), drug resistance.

\section{Introduction}

According to global Tuberculosis (TB) reports, TB has been one of the leading causes of the burden on the health care systems and economy due to the massive number of cases recorded per year as stated by global TB report. This led to the initiation of a target goal by 2030 with the title "End TB Strategy" by the world health organization and the united nation. It aims to reduce morbidity in TB by $90 \%$ and reduce morbidity incidence by $80 \%$ in comparison to the year 2015. [1].

Despite the appreciable renovation in the notification and prosperous TB management protocol in Saudi Arabia, a moderate infection rate is still reported compared with other countries [2]. The wavering TB Prevalence over the kingdom with diverseness across its regions is well recognized in the recent local studies [3]. This demonstrates why western provinces, particularly Makkah province, for instance, in Jeddah (Sea and Airports for pilgrims arriving into Makkah), have the preeminent TB incidence in the country [4]. 
This is explained by the fact that Makkah is the holy place for Muslims and is visited annually by millions of pilgrims from countries where TB is highly endemic.

The relatively high burden of TB in Saudi Arabia necessitates Better reviews to ease the utilization of proper and rapid diagnostics and typing methodologies to maximize treatment efficacy.

This study aimed to determine the status of TB in Jeddah, the age and the gender distribution, the accuracy of conventional diagnostic methods, the rates and the patterns of anti-TB drug resistance, all in correlation with the primary site of infection and in comparison, with national and international figures.

\section{Materials and Methods}

The study was conducted at an internationally accredited tertiary care center in Jeddah, Saudi Arabia, which is a 300-bed tertiary care hospital. The duration of the study was spanned over 10 years, starting from the year 2006, and was approved by the King Faisal Specialist Hospital and Research Centre Institutional Review Board (IRB 2016-27).

\subsection{Clinical Specimens}

In this study, a total of 345 pulmonary and extrapulmonary samples obtained during the clinical evaluation of the patients and were sent for investigation in the Mycobacteriology Laboratory between November 2006 to August 2016. The 254 samples included in the study were NAAT positive isolates for mycobacterium tuberculosis complex.

135 samples were pulmonary and 119 were extrapulmonary specimens. The pulmonary specimens were obtained from (sputum, tracheal aspirate, bronchoalveolar lavage, pleuritic fluid, and lung tissue), and the extrapulmonary specimens were collected from (cerebrospinal Fluid, blood, lymph node, gastric fluid, drain fluid, bone specimen, tissue specimen and abscess).

\subsection{Specimens Processing and Culturing:}

Nonsterile clinical specimens were processed by the conventional N-acetyl-L-Cystein-Sodium-hydroxide $(\mathrm{NaLC}-\mathrm{NaOH})$ method [5]. After which the smears were decontaminated, then prepared by the auraminerhodamine acid-fast staining method. Decontaminated smears were inoculated to Lowenstein-Jensen (LJ) solid medium and Growth Indicator Tubes (Bactec, MGIT 960, Becton Dickinson, Franklin Lakes, NJ, USA) liquid media for growth detection, and the leftover sediment of the decontaminated specimen was stored at $-70 \mathrm{C}^{\mathrm{o}}$. Cultures that have failed to produce a positive result within six weeks were labeled negative of mycobacterium tuberculosis complex $(M T B C)$ infection. Kinyoun stain was applied for all positive cultures, MTBC strains growth on MGIT medium were tested for drug susceptibility using previously described methods [6].

All types of clinical samples were cultured in one liquid medium containing Middlebrook 7H9 broth base (MGIT, Becton-Dickinson (BD), Heidelberg, Germany) after they have been processed by NaLC-NaOH. For NAAT testing, BD ProbTech ET system for MTBC (Becton Dickinson, Franklin lakes, NJ, USA) was used. NAAT positive isolates for MTBC (254 isolates) were analyzed statistically and analyzed using SPSS. All tubes had been monitored continuously until yielding positive results or until reaching the end of the testing period (after six weeks). The mean number of days for the cultures to become positive was 12.79, with a minimum value of 4 and a maximum of 64 days.

\section{Results}

A total of 345 patients with a positive culture were enrolled in this study. $67.8 \%$ were positive for mycobacterium tuberculosis complex, $24.1 \%$ were positive for non-mycobacterium tuberculosis, $0.9 \%$ were positive for mycobacterium tuberculosis mixed with non-mycobacterium tuberculosis, with the remaining $7.2 \%$ being negative. Of the positive cultures for Mycobacterium tuberculosis complex, $99.1 \%$ were positive using NAAT. From the NAAT positive samples, only $36.8 \%$ were positive for AFB, while the remaining $63.8 \%$ were negative for AFB.

Among the positive mycobacterium tuberculosis complex cases, the mean patient age was 51 years $(4.7 \%$ were less than 14 years, $59.4 \%$ between the ages of 14 and $60,35.8 \%$ were 60 years or older). Of these 
samples, $56.3 \%$ were from males, while $43.7 \%$ were from females' patients. Pulmonary specimens constituted $53.1 \%$ of the samples, and $46.9 \%$ were from extrapulmonary sites of infection.

When the susceptibility of 1st line anti-TB agents were tested on the positive samples, $10.2 \%$ Showed Resistance to Isoniazid, $2.4 \%$ to Rifampicin, $1.2 \%$ to Ethambutol, $5.1 \%$ to pyrazinamide, and $11.0 \%$ to streptomycin.

There was a difference in drug resistance when pulmonary tuberculosis (PTB) samples were compared with the extrapulmonary tuberculosis (EPTB) samples, in form of $(8.9 \%-11.8 \%)$ to Isoniazid , $(1.5 \%-3.4 \%)$ to rifampicin , $(0.7 \%-1.7 \%)$ to ethambutol,$(4.4 \%-5.9 \%)$ to pyrazinamide and $(12.6 \%-9.2 \%)$ to streptomycin, for PTB and EPTB, respectively. However, this difference was not statistically significant in our study - P-Value > 0.05 [Table-1].

Table-1: First line anti-tuberculosis agents resistance

\begin{tabular}{|l|l|l|l|l|l|}
\hline & \multicolumn{2}{l|}{} & \multicolumn{2}{l|}{$\begin{array}{l}\text { Anti-tuberculosis agents resistance } \\
\text { According to the site of infection }\end{array}$} \\
\hline Anti-Tuberculosis Agent & Resistance & P Value & Pulmonary & Extrapulmonary & P Value \\
\hline Isoniazid & $10.2 \%(26)$ & $<0.05$ & $8.9 \%(12)$ & $11.8 \%(14)$ & 0.717 \\
\hline Rifampicin & $2.4 \%(6)$ & $<0.05$ & $1.5 \%(2)$ & $3.4 \%(4)$ & 0.582 \\
\hline Ethambutol & $1.2 \%(3)$ & $<0.05$ & $0.7 \%(1)$ & $1.7 \%(2)$ & 0.738 \\
\hline Pyrazinamide & $5.1 \%(13)$ & $<0.05$ & $4.4 \%(6)$ & $5.9 \%(7)$ & 0.823 \\
\hline Streptomycin & $11.0 \%(28)$ & $<0.05$ & $12.6 \%(17)$ & $9.2 \%(11)$ & 0.595 \\
\hline Multi Drug Resistance & $2.4 \%(6)$ & $<0.05$ & $1.5 \%(2)$ & $3.4 \%(4)$ & 0.325 \\
\hline
\end{tabular}

Our study showed that $1.3 \%$ of our patients were resistant to all first-line anti-TB agents, $2.2 \%$ to three of them, $3.9 \%$ to two agents, and $11.6 \%$ to one agent, while $81.0 \%$ were sensitive to all agents. MDR TB, which is defined as TB that does not respond to at least isoniazid and rifampicin, the 2 most powerful antiTB drugs, was found in $2.4 \%$ of our patients. MDR TB was found more in samples that were taken from the extrapulmonary site of infection compared to the pulmonary site $(3.4 \%, 1.5 \%$, respectively).

\section{Discussion}

Published reports from Saudi Arabia in 2010 revealed that the elderly population was the dominant age group affected by $\mathrm{TB}$, which was seemingly attributed to reactivation of latent $\mathrm{TB}$, as researches revealed a higher prevalence of Tuberculin skin test positivity during the early 1990s [7]. In contrast, our study showed that TB has been more common in the younger generation by $(59.4 \%)$, which is principally referred to the accelerated rise of the human immunodeficiency virus (HIV) in the country, as the WHO reported that $51 \%$ of TB in Saudi Arabia was found most amongst positive HIV status [8]. A study conducted on the data collected over 10 years demonstrated that the incidence rate of TB in people living with HIV in Saudi Arabia is 30 folds surpassing the general population rate [9], taking into consideration, that there is a high number of young foreign worker recruitment in the endemic areas with TB, which may contribute to such distribution [10]. Fortunately, TB amongst the pediatric population is $4.7 \%$ which can be credited to the high level of Bacillus-Calmette-Guerin (BCG) vaccine coverage, which is obligatory in the kingdom and approaching approximately $97.5 \%$ of the newborn [11].

Direct sputum smear is the most universally used method for diagnosis of TB because of its high specificity, but unfortunately, it has variable sensitivity ranging between $20 \%$ to $80 \%$ [12]. Which can be best noticed In countries with moderate to the high prevalence of both PTB and HIV infection, where the false-negative rate is higher due to the paucibacillary nature of TB which is described in earlier diagnostic studies [13], and greatly demonstrated in our study, with only $36.8 \%$ of culture-proven TB being positive by direct smear.

Compared with smear microscopy, the NAAT has an extra value which can be acknowledged in multiple aspects. Determined by Its higher positive predictive value (PPV) (>95\%) for TB with AFB smear-positive samples in a context where Non mycobacterium tuberculosis are of considerable percentage $(24.1 \%$ in our study), as well as its capability to promptly verifies the presence of Mycobacterium tuberculosis in $50 \%$ to $80 \%$ of AFB negative, culture-positive specimens (63.8\% in our study). When properly implemented, the NAAT will boost the diagnostic efficiency in cases of complicated infections that are suspected to be TB, with subsequent advancement in time and cost-effective patient care [12,14]. 
About $35.8 \%$ of our culture-proven TB was taken from patients ages 65 and above. TB presentation in a senior adult could be atypical, and may not exhibit the traditional manifestations of TB, which may alter the detection rate of TB and physicians should have a high level of suspicion when dealing with this age group in order to recognize TB in its atypical presentation [15]. Gender distribution in our study was similar to the global TB pattern [1], with slightly male predominance, which could be attributed to the exposure factors [10].

Referring to the national reports recorded EPTP percentage since 1989, the initial period showed substantial decrement, followed by a noticeable gradual increase in the incidence rate of EPTP, best demonstrated by the reported percentage $(11.7 \%)$ of EPTP in 1991, with a sharp increase to $(28.2 \%)$ in 1997 , with an afterward consistent level of (30\%) reported annually from national TB notification registry data [16]. Our study showed an even higher incidence level (46.9\%) of cases) [16]. The diagnosis of EPTB is often challenging as patients are more liable to have negative sputum smear results as the absence of direct lung involvement is not unusual and may obligate tissue sampling from the suggested site of infection to document the diagnosis by histopathology or microbiology [17].

Mono resistance to Streptomycin $11.0 \%$, was the highest among our study, followed by isoniazid $10.2 \%$, pyrazinamide $5.1 \%$, Rifampicin $2.4 \%$, then Ethambutol $1.2 \%$. However, anti-TB agents' mono resistance had been declining in studies done in 2010 compared to studies done fifteen years earlier in 1995 [18], but unfortunately, the trend of resistance started to rise again, as shown in our study and illustrated in [Figure 1].

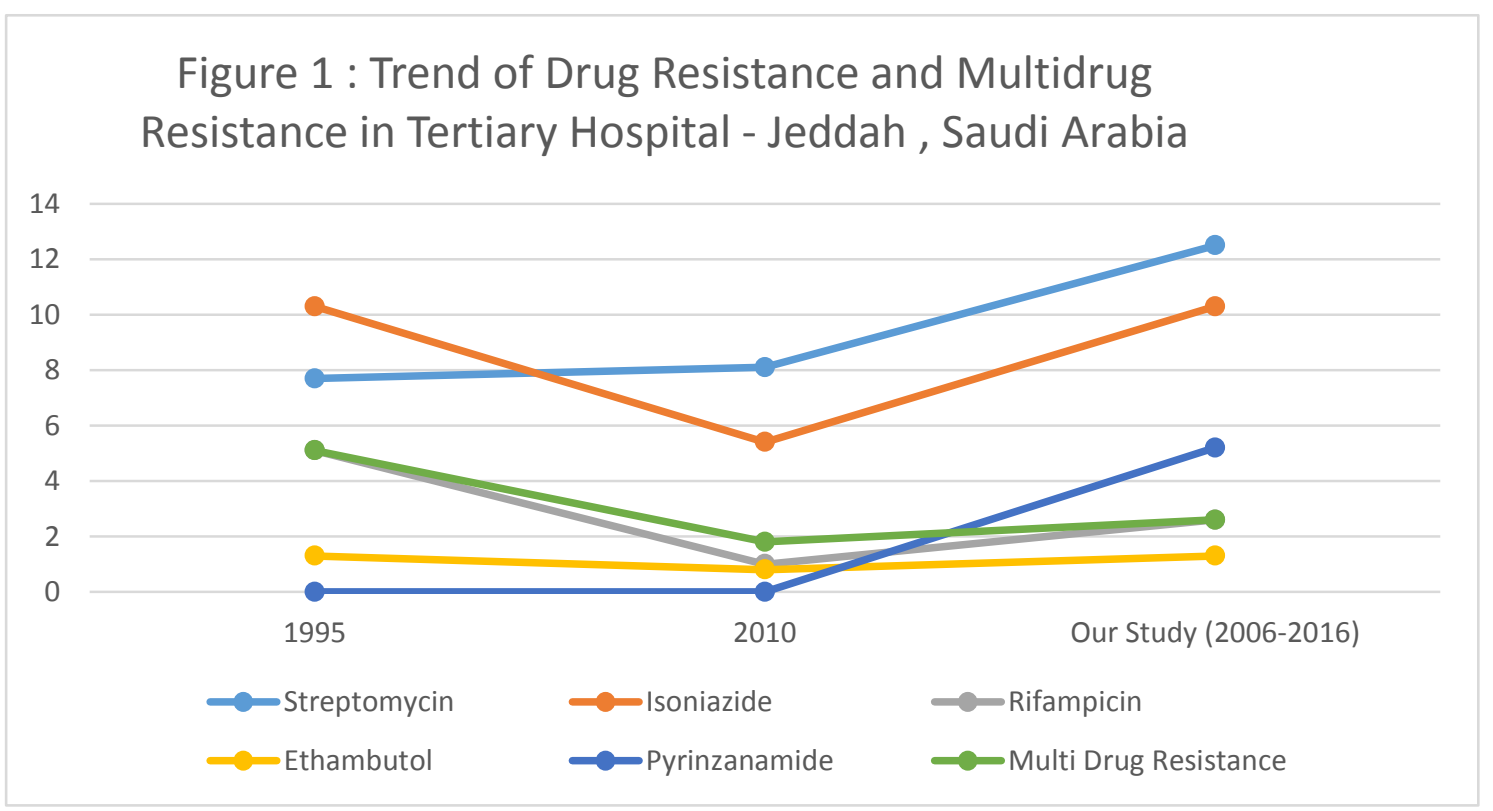

In comparison to a systematic review, that was published in 2015 which included 22 studies from Saudi Arabia published between the years 1979 and 2013 [19], our data showed a similar range in the resistance pattern to isoniazid and ethambutol but a rise in streptomycin resistance coupled with a reduction in rifampicin resistance. However, the resistance trend for pyrazinamide lacked in most of the studies done in Saudi Arabia.

The rate of drug-resistant TB in Saudi Arabia is largely variable between the kingdom's provinces in the published articles, which might be mainly influenced by different geographic locations, as well as the percentage of non-Saudi residents, especially with the presence of ex-pats who came from TB Endemic areas [20].

Streptomycin resistance was highest among the anti-TB medications investigated in our study, the rationale behind this relatively high percentage, which is also reported in other national and international studies is the presence of the genetic mutation in the bacilli cell that alter the streptomycin binding site, which was found in approximately half of the isolates studied across the world [21]. 
Although international studies described genetic mutations being behind TB Resistant to other first-line agents, studies from Saudi Arabia attributed the increasing resistance rate mainly to non-adherence with medication, which subsequently might have led to treatment failure, with rifampicin resistance being the strongest predictor to develop MDR, while ethambutol resistance is least statistically significant associated with MDR [22,23]

Unlike PTB, there is no reliable data accurately reporting the systemic drug resistance for EPTB in most national and international studies, as many of the high endemic rates are resource-limited and not routinely perform susceptibility testing for EPTB. However, sporadic information derived from retrospective cohort studies and TB surveillance data showed that the chance of getting a mono anti-tuberculous drug resistance at any time is about $10 \%-75 \%[24]$.

Although there was no statistically significant difference between resistance to any first-line anti-TB drug in PTB (19.3\%) compared to EPTB (19.0\%), the sub-analysis of drug resistance profile showed a higher proportion of resistance in EPTB compared to PTB, and similar findings were observed in studies adopted from India and Taiwan, which are high endemic areas of TB [24].

MDR TB percentage in our study was $2.4 \%$, almost similar to WHO global statistics for Saudi Arabia (2.6\%). Although initially declined to level of $1.8 \%$ as confirmed in national studies reported the time interval between 1990 and 2010 , MDR TB started to re-emerge afterward as documented in our study and other recent national systemic review - studies among 22 hospitals in different regions of Saudi Arabia , reporting an increasing level of $2.4 \%$ and $6.7 \%$ respectively $[1,19,20]$.

Multiple factors play a role in the MDR rate variation between Saudi regions, including, but not limited to the nature of patients included in the study, i.e. newly or previously treated, as well as the time interval of the study. However, reasonably, several studies revealed that the western region has the highest proportion of MDR TB as it has the largest number of foreigner ex-pats [25].

The MDR in the EPTB isolates was found to be lower than MDR in PTB isolates $(1.1 \%-2.6 \%)$. This result was similar to the findings of a retrospective study published in 2015 analyzing culture-positive EPTB isolates collected from all the provinces of Saudi Arabia with MDR of (2.1\% - 4.5\%) for PTB and EPTB respectively [26]. According to another international study simultaneously done in 2015, suggested it may be attributed to more selective multiplication of resistant mutants of Mycobacterium tuberculosis in cavitary lesions in the lung in comparison to caseous focal lesions of extrapulmonary sites [24].Other factors that contribute to the development of resistance in EPTB may include late diagnosis, inappropriate anti-TB regimen prescription or poor patient's adherence to a proper anti-TB regimen [27].

\section{Conclusion}

Our study was based on retrospective data collected from a tertiary hospital in Jeddah, Saudi Arabia which was spanned over ten years. The results showed that TB was predominant in the younger age group by $59.4 \%$ in comparison to other populations which may be attributed to the high influx of workers from endemic regions and increasing co-infections with HIV. The other age groups were less in comparison due to mandatory immunization in the pediatric age group, and atypical presentation of the elderly population.

The golden standard to diagnose TB is by culture-based method, whenever possible, and due to high positivity rate $(91.1 \%)$ NAAT should be obtained to establish the diagnosis, especially with low positivity rate of AFB smear in our country $(36.8 \%)$ and to differentiate mycobacterium tuberculosis from nonmycobacterium tuberculosis, which is of considerable percentage in our country $(24.1 \%)$.

Re-emergence of TB resistance was observed in our study compared to previous national surveys carried between 1995 and 2010, with resistance level of $11.0 \%, 10.2 \%, 5.1 \%, 2.4 \%, 1.2 \%, 2.4 \%$ to Streptomycin, isoniazid, pyrazinamide, Rifampicin, and Ethambutol and MDR respectively. 
Our study acknowledged a higher proportion of mono drug resistance in EPTB in comparison to PTB. Necessitating the physician's awareness when treating patients with EPTB. Nevertheless, this finding was not statistically significant and may need further extended studies.

This study has few limitations, newly diagnosed TB cases could not be compared with previously infected cases, the immune status of the patient (i.e. Co infection with HIV or previous malignancy) was not known to us during the study. Finally, King Faisal Specialist Hospital being a referral center, made us not able to investigate all patients with different nationalities, environment, and socioeconomic backgrounds.

\section{References}

[1.] Global tuberculosis report 2016, world health organization, Retrieved from http://www.who.int/tb/publications/global_report/gtbr2016_main_text.pdf

[2.] Al-Hajoj, S. A. (2010). Tuberculosis in Saudi Arabia: Can we change the way we deal with the disease? Journal of Infection and Public Health, 3(1), 17-24. doi:10.1016/j.jiph.2009.12.001

[3.] Al-Hajoj, S., Varghese, B., Shoukri, M. M., Al-Omari, R., Al-Herbwai, M., Alrabiah, F., . . . Memish, Z. (2013). Epidemiology of Antituberculosis Drug Resistance in Saudi Arabia: Findings of the First National Survey. Antimicrobial Agents and Chemotherapy, 57(5), 2161-2166.

[4.] Yezli, S., \& Memish, Z. A. (2012). Tuberculosis in Saudi Arabia: prevalence and antimicrobial resistance. Journal of Chemotherapy, 24(1), 1-5.

[5.] Al-Orainey, I., Alhedaithy, M., Alanazi, A., Barry, M., \& Almajid, F. (2013). Tuberculosis incidence trends in Saudi Arabia over 20 years: 1991-2010. Annals of Thoracic Medicine,8(3), 148.

[6.] [6]. Kent. P. T., and G.P. Kubica (1985). Public health mycobacteriology. A guide for a level III laboratory. Centers for Disease Control, Atlanta, GA.

[7.] Bemer P, Palicova F, Rüsch-Gerdes S, Drugeon HB, Pfyffer GE. (2002). Multicenter evaluation of fully automated BACTEC Mycobacteria Growth Indicator Tube 960 system for susceptibility testing of Mycobacterium tuberculosis. Journal of Clinical Microbiology, 40:150-154.

[8.] Tuberculosis country profiles. (Saudi Arabia). Retrieved July 15, 2017, from http://www.who.int/tb/country/data/profiles/en/

[9.] Al-Hajoj, S., \& Varghese, B. (2015). Tuberculosis in Saudi Arabia: the journey across time. The Journal of Infection in Developing Countries, 9(03), 222.

[10.] Abouzeid, M. S., Zumla, A. I., Felemban, S., Alotaibi, B., O'Grady, J., Memish, Z. A. (n.d.). Tuberculosis Trends in Saudis and Non-Saudis in the Kingdom of Saudi Arabia - A 10 Year Retrospective Study (2000-2009).

[11.] Al-Shehri, S. N., \& Al-Shammari, S. A. (1991). Immunization coverage survey in eight regions of Saudi Arabia. Annals of Tropical Paediatrics, 11(2), 181-187.

[12.] Ryu YJ. (2015). Diagnosis of pulmonary tuberculosis: recent advances and diagnostic algorithms. Tuberc Respir Dis (Seoul), 78(2):64-71.

[13.] Siddiqi K, Lambert ML, Walley J. (2003). Clinical diagnosis of smear-negative pulmonary tuberculosis in low-income countries: the current evidence. Lancet Infect Dis, 3(5):288-96.

[14.] Salian NV, Rish JA, Eisenach KD, Cave MD, Bates JH. (1998). Polymerase chain reaction to detect Mycobacterium tuberculosis in histologic specimens. Am J Respir Crit Care Med, 158(4):1150-5.

[15.] Rajagopalan, S. (2001). Tuberculosis and Aging: A Global Health Problem. Clinical Infectious Diseases, 33(7), 1034-1039.

[16.] Varghese, B., \& Al-Hajoj, S. (2015). Mapping the epidemiology and trends of extra-pulmonary tuberculosis in Saudi Arabia. International Journal of Mycobacteriology, 4(4), 261-269.

[17.] Purohit M, Mustafa T. (2015). Laboratory Diagnosis of Extra-pulmonary Tuberculosis (EPTB) in Resource-constrained Setting: State of the Art, Challenges and the Need. J Clin Diagn Res, 9(4):EE01-6.

[18.] Abu-amero KK. (2002). Status of antituberculosis drug resistance in Saudi Arabia 1979-98. East Mediterr Health J, 8(4-5):664-70. 
[19.] Al-Hajoj, S., et al. (2013). Epidemiology of Antituberculosis Drug Resistance in Saudi Arabia: Findings of the First National Survey. Antimicrobial Agents and Chemotherapy, 57(5): 21612166.

[20.] Al-Tawfiq, J. A., Hinedi, K., \& Memish, Z. A. (2015). Systematic review of the prevalence of Mycobacterium tuberculosisresistance in Saudi Arabia. Journal of Chemotherapy, 27(6), 378-382.

[21.] Silva, P. E., \& Palomino, J. C. (2011). Molecular basis and mechanisms of drug resistance in Mycobacterium tuberculosis: classical and new drugs. Journal of Antimicrobial Chemotherapy, 66(7), 1417-1430.

[22.] Samman, Y., Krayem, A., Haidar, M., Mimesh, S., Osoba, A., Al-Mowaallad, A., Wali, S. (2003). Treatment outcome of tuberculosis among Saudi nationals: role of drug resistance and compliance. Clinical Microbiology and Infection, 9(4), 289-294.

[23.] Kim YH, Suh GY, Chung MP, et al. Treatment of isoniazid-resistant pulmonary tuberculosis. BMC Infect Dis. 2008;8:6.

[24.] Singh, P. K. (2015). Epidemiological perspective of drug resistant extrapulmonary tuberculosis. World Journal of Clinical Infectious Diseases, 5(4), 77.

[25.] Ahmed, M. M., Velayati, A. A., \& Mohammed, S. H. (2016). Epidemiology of multidrug-resistant, extensively drug resistant, and totally drug resistant tuberculosis in Middle East countries. International Journal of Mycobacteriology, 5(3), 249-256.

[26.] Al-Hajoj, S., Shoukri, M., Memish, Z., Alhakeem, R., Alrabiah, F., \& Varghese, B. (2015). Exploring the Sociodemographic and Clinical Features of Extrapulmonary Tuberculosis in Saudi Arabia. Plos One, 10(2).

[27.] Xu L, Jian-zhong X, Xue-mei L, Bao-feng G. (2013). Drug susceptibility testing guided treatment for drug-resistant spinal tuberculosis: a retrospective analysis of 19 patients. Int Surg, 98(2):17580 . 